\title{
Case Report \\ Scleral Rupture Secondary to Idiopathic Non-Necrotizing Scleritis in a Dog
}

\author{
Lori J. Best, Shelley J. Newman, Daniel A. Ward, and Diane V. H. Hendrix
}

University of Tennessee, 2407 River Drive, Knoxville, TN 37919, USA

Correspondence should be addressed to Daniel A. Ward; dward@utk.edu

Received 26 March 2013; Accepted 21 April 2013

Academic Editors: C. Hyun, J. Lakritz, F. Mutinelli, and J. Orós

Copyright (C) 2013 Lori J. Best et al. This is an open access article distributed under the Creative Commons Attribution License, which permits unrestricted use, distribution, and reproduction in any medium, provided the original work is properly cited.

\begin{abstract}
Background. Canine granulomatous scleritis is an uncommon disease that can be classified as necrotizing or non-necrotizing. Clinical signs associated with scleritis are typically severe, resulting in pain and loss of vision, and response to treatment is often poor. Necrotizing scleritis has been previously associated with scleral rupture. Case Presentation. A 10-year-old male castrated Chihuahua was presented for periocular pain, tissue swelling adjacent to the limbus superiorly, chemosis, mild corneal edema and neovascularization adjacent to the superotemporal limbus in the right eye. The left eye was within clinically normal limits. Surgical exploration of the right eye revealed a scleral rupture at the inferonasal aspect of the globe. Histopathology revealed a nonnecrotizing granulomatous scleritis with no infectious organisms visualized. Infectious disease testing and special histopathologic staining did not reveal an underlying infectious etiology. Conclusion. Granulomatous scleritis is a painful and vision-threatening disease that needs to be treated early and aggressively in order to avoid loss of vision or loss of the eye. Globe rupture secondary to severe non-necrotizing scleritis is an uncommon, but detrimental, clinical manifestation of this disease. This is the first case report of scleral rupture secondary to severe non-necrotizing scleritis and therefore represents a unique and interesting disease manifestation.
\end{abstract}

\section{Background}

Scleritis is an uncommon and poorly understood disease process in dogs [1]. The sclera comprises approximately $80 \%$ of the fibrous outer portion of the eye and is the posterior continuation of the transparent cornea. The sclera is closely associated with several ocular tissues and for this reason secondary keratitis, conjunctivitis, chorioretinitis, orbital cellulitis, and blepharitis may be seen in cases of primary scleritis [2]. The etiology of canine scleritis is often presumed to be immune-mediated due to the characteristic presence of granulomatous inflammatory infiltrates, response to immunosuppressive therapy, and inability to identify microorganisms by histopathology [3]. Other potential underlying causes of scleritis include Ehrlichia canis, Onchocerca spp., Toxoplasma gondii, trauma (including surgical trauma), and extension of panophthalmitis or orbital cellulitis $[1,3,4]$. In humans, granulomatous scleritis is often associated with systemic vascular or autoimmune collagen disorders including rheumatoid arthritis [5], Wegener's granulomatosis [6], and systemic lupus erythematosus [7]. Some reported cases of scleritis in dogs have tested negative for canine rheumatoid factor, antiDNA antibody, and LE cell identification, and there has not been a clearly established link between scleritis and systemic autoimmune disease in dogs [8].

Clinical signs associated with canine scleritis include ocular hyperemia, ocular pain, and exophthalmos, as well as clinical signs associated with secondary anterior uveitis, posterior uveitis, conjunctivitis, or keratitis. Clinical signs are frequently unilateral upon initial admission but the disease will often progress to affect the other eye [9]. A definitive diagnosis of scleritis can be achieved histologically with a partial thickness biopsy or after enucleation [10]. Canine scleritis is not well described in the peer-reviewed literature, so it is difficult to accurately determine prognosis. In one series canine scleritis cases showed little improvement with local and systemic immunosuppressive treatment and the prognosis for affected eyes was considered guarded to poor [1]. 


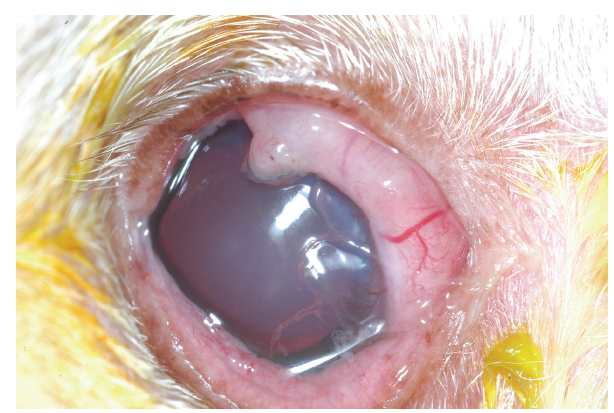

Figure 1: Appearance of right eye on initial presentation. Notice fluctuant swelling of the conjunctiva. There is also mild corneal edema and neovascularization.

Histologically, canine scleritis is characterized by a diffuse granulomatous cell infiltrate composed predominantly of tissue macrophages, lymphocytes and plasma cells. In severe cases, the inflammatory cells may completely efface normal scleral tissue [1]. Granulomatous scleritis is further categorized as necrotizing or non-necrotizing based on whether or not there is lysis or merely separation of scleral collagen [9]. In cases of chronic scleritis, thinning of the scleral collagen with subsequent severe staphyloma formation can occur [9]. One review suggests that the scleral degeneration seen with necrotizing scleritis can result in scleral malacia and perforation [11]. Necrotizing scleritis and scleral rupture have been associated with Ehrlichia canis [12].

The purpose of this report is to highlight the clinical and histopathological features of a case of presumed immunemediated non-necrotizing granulomatous scleritis in a dog. This is a unique presentation of the disease because of the associated scleral rupture, and has not been previously reported to the authors' knowledge.

\section{Case Presentation}

A 10-year-old male castrated Chihuahua was presented to the University of Tennessee College of Veterinary Medicine ophthalmology service for evaluation of blepharospasm, mucoid ocular discharge, and progressive swelling of the superior conjunctiva of the right eye (OD) (Figure 1). There was an approximately 1 week history of blepharospasm, epiphora, chemosis, corneal edema, and a pale yellow nodule on the superonasal conjunctiva that was being treated three times daily with bacitracin/neomycin/polymyxin B/dexamethasone ophthalmic ointment (BNP-Dex) OD. The corneal edema and conjunctival nodule appeared to resolve with BNP-Dex treatment. Diarrhea was noticed by the owners at approximately the same time as the development of blepharospasm. The dog was exposed to a lemur that had been diagnosed with Yersinia pseudotuberculosis, had no previous history of ophthalmic or systemic disease, and had no significant travel history.

There were no abnormalities on general physical examination aside from a mild fever of $39.4^{\circ} \mathrm{C}$. On ophthalmic examination, pupillary light reflexes and menace responses were normal in both eyes (OU). Schirmer tear tests and

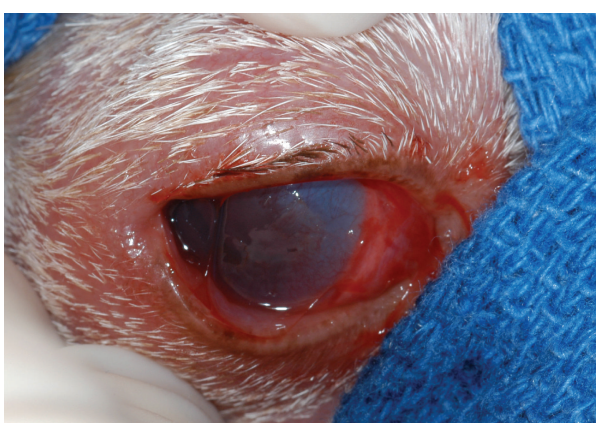

FIGURE 2: Appearance of right eye 2 weeks after initial presentation. Notice severe periorbital swelling, conjunctival hyperemia, and corneal edema and neovascularization.

intraocular pressures were within normal limits $\mathrm{OU}$ and both eyes were negative for fluorescein stain uptake. The left eye (OS) was normal on slit lamp biomicroscopy and indirect ophthalmoscopy. The following abnormalities were observed in the OD: moderate blepharospasm, mucoid ocular discharge, pain on palpation of the periorbital tissues, conjunctival swelling and chemosis, mild corneal edema and neovascularization adjacent to the superotemporal limbus, and mild aqueous flare. The lens, vitreous and fundus were within normal limits OD. Based on the ocular exam findings, an ocular ultrasound was recommended.

Ultrasonographic images were obtained using a $15 \mathrm{MHz}$ linear array probe. An area of hypoechogencity, possibly representing fluid accumulation or a cyst, was identified adjacent to the ventral limbus OD. Potential differential diagnoses for this structure were staphyloma, occult scleral rupture, or conjunctival foreign body with surrounding seroma formation. Based on these findings, periocular exploratory surgery was recommended. The owner declined surgical intervention and opted to continue therapy with BNP-Dex.

The patient was presented 2 weeks later for a recheck examination. At that time a fever of $40.4^{\circ} \mathrm{C}$ was present and the OD was markedly more uncomfortable compared to the initial visit. The left eye was normal. Severe periorbital swelling and pain were identified OD (Figure 2). Midstromal neovascularization and severe diffuse corneal edema were present in the right cornea, and moderate aqueous flare was present within the anterior chamber OD.

Based on the worsening clinical course, lack of response to treatment, and with differentials that included surgically treatable lesions, the owner consented to surgical exploration of the right periorbital region.

A complete blood cell count, serum biochemistry panel with electrolytes, and urinalysis were submitted prior to anesthesia. The complete blood cell count revealed a neutrophilia of $15.29 \times 10^{3} / \mathrm{uL}$ (RR: $2.65-9.8 \times 10^{3} / \mathrm{uL}$ ) and increased band neutrophils of 550/uL (RR: 0-300/uL) consistent with an inflammatory leukogram. A mild hypochromic microcytic anemia was present with a hematocrit of $39.9 \%$ (RR: $41 \%-60 \%$ ), MCHC of $30.3 \mathrm{~g} / \mathrm{dL}$ (RR: $34.5-36.3 \mathrm{~g} / \mathrm{dL}$ ), and MCV of $59.2 \mathrm{fL}$ (RR: $62-74 \mathrm{fL}$ ). The serum biochemistry panel revealed an increased total protein of $9.5 \mathrm{~g} / \mathrm{dL}$ (RR: $5.4-6.8 \mathrm{~g} / \mathrm{dL}$ ) with a decreased albumin of $2.0 \mathrm{~g} / \mathrm{dL}$ (RR: 


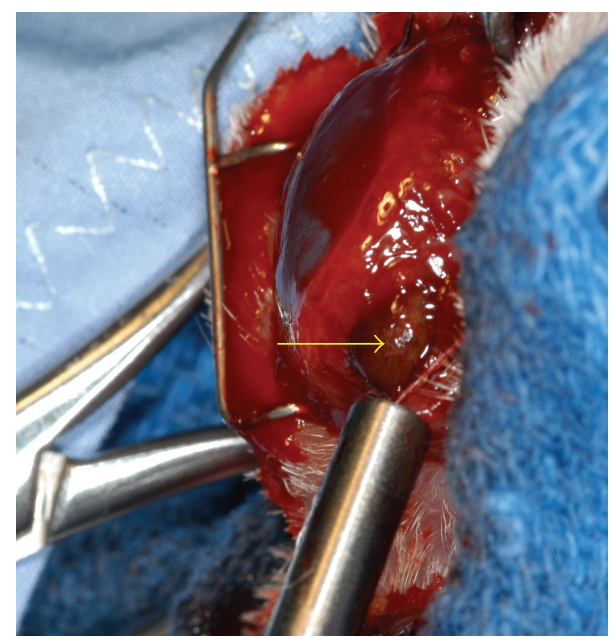

FIGURE 3: Intraoperative appearance. The conjunctiva has been dissected away and uveal tissue can be seen prolapsing through the region of scleral rupture (yellow arrow).

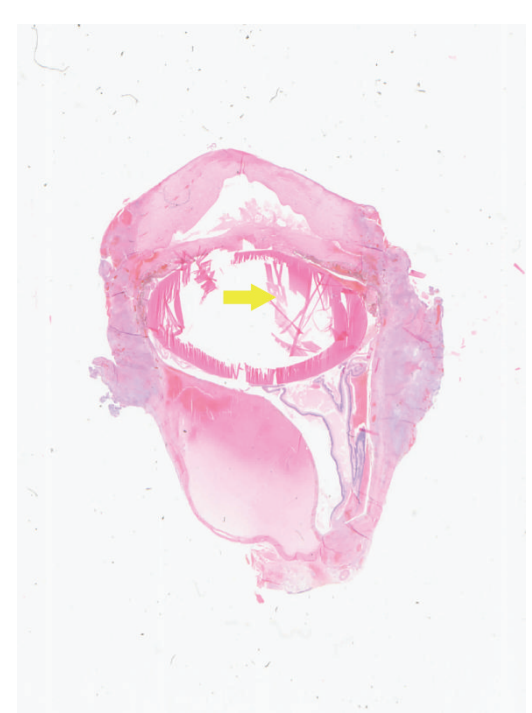

Figure 4: Low power view of the globe. The lens is artifactually fragmented (arrow). There is a marked inflammatory response centered at the limbus. In addition, there is retinal detachment and accumulation of fluid in the subretinal space. The cornea is markedly thickened and there is early keratitis.

3.2-4.1 g/dL) and an increased globulin of $7.5 \mathrm{~g} / \mathrm{dL}$ (RR: 2$3.2 \mathrm{~g} / \mathrm{dL}$ ). Protein electrophoresis indicated polyclonal gammopathy, indicative of chronic antigenic stimulation.

The patient was placed in sternal recumbency and the right eye was sterilely prepped and draped. A $1 \mathrm{~cm}$ lateral canthotomy was made with tenotomy scissors and a 60 silk stay suture was placed at the superior limbus. The superior bulbar conjunctiva was then incised approximately $2 \mathrm{~mm}$ posterior to the limbus with curved tenotomy scissors and the conjunctiva bluntly dissected with tenotomy scissors while the bulb was rotated inferiorly. Hemorrhagic chemosis, sclerosis, and pockets of fibrinonecrotic material

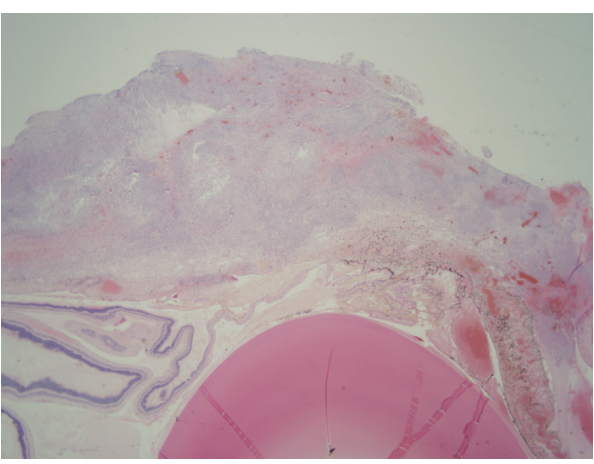

FIGURE 5: At the limbus, a markedly severe multinodular inflammatory infiltrate is present within and expanding from the limbus.

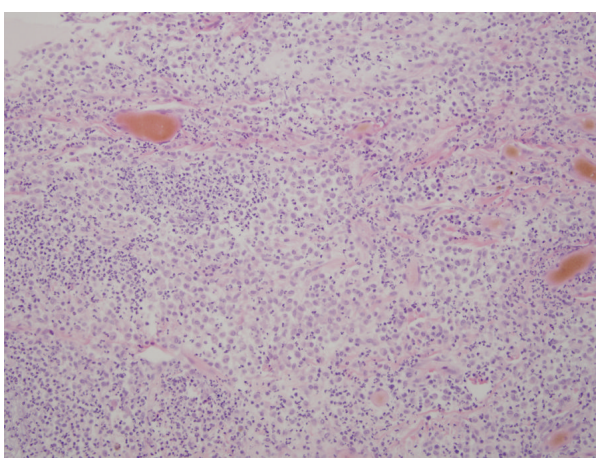

FIGURE 6: The inflammatory infiltrate is distinctive in that it is composed of recurring clusters of neutrophils surrounded by reactive foamy macrophages (pyogranulomas) which coalesce to create the limboscleral inflammation.

were encountered throughout the conjunctiva. The periorbital musculature was also noted to be sclerotic. No cystic structures or foreign bodies were encountered superiorly, so exploration was continued nasally and inferonasally. Upon examination of the inferonasal aspect of the globe, a scleral rupture was found at the 4 o'clock position, approximately $3 \mathrm{~mm}$ posterior to the limbus (Figure 3). Uveal tissue was herniated through the rupture, and vitreous humor and aqueous humor were leaking from the site. The sclera surrounding the rupture appeared malacic. An attempt to close the scleral rupture was made with 8-0 polyglactin 910 but there was not sufficient normal scleral tissue present to allow suturing. The surgery was then converted to a standard subconjunctival enucleation. The globe was placed in Davidson's solution for fixation and submitted for histopathologic evaluation.

A histologic section of the enucleated globe was made via sagittal section through the entire eye. The tissue sample was stained with hematoxylin and eosin (H\&E) and with Masson's trichrome. There was a severe diffuse granulomatous scleritis present, more concentrated at the level of the limbus (Figures 4 and 5). The inflammation was characterized primarily by macrophages that were arranged in aggregates and sheets and accompanied by lymphocytes and fewer neutrophils (Figure 6). In perilimbal regions, the granulomatous infiltrate was more marked and the sclera had an 


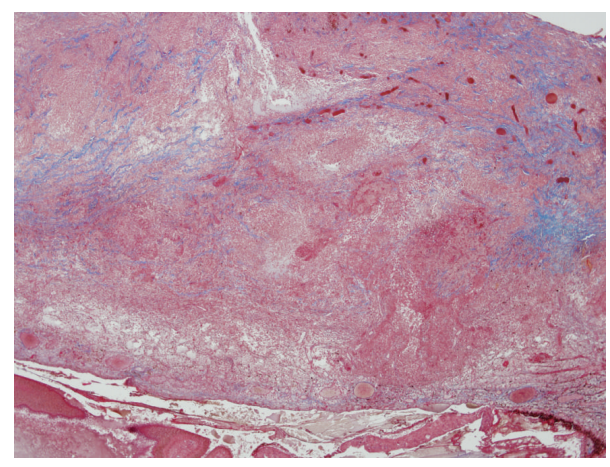

FIgURE 7: Sclera at the limbus. In this Masson trichrome stained section, there is marked separation of collagenous stroma (blue) by the eosinophilic cellular infiltrate. The collagen is not necrotic.

increased thickness, as a result of separation of intact collagen bundles by cellular infiltrate (Figure 7). The corneal stroma was diffusely expanded by edema and there were multifocal small caliber vessels (neovascularization) and scattered neutrophils. The anterior chamber contained red blood cells, neutrophils, epithelioid macrophages, multinucleated giant cells, and fibrin. There was regionally extensive separation of the choroid from the sclera and the cleft was filled with red blood cells and fibrin. The retina was diffusely detached and there was hypertrophy of the retinal pigment epithelium (RPE). Hemorrhage, fibrin, and karyorrhectic debris filled the space separating the retina and RPE. The vitreous chamber contained fibrin and karyorrhectic debris.

Periodic acid-Schiff (PAS) and GMS stains failed to reveal fungi or yeasts and Ziehl-Neelsen ( $\mathrm{Zn})$ and Fite's failed to reveal mycobacteria or atypical mycobacteria. A 16sRNA test performed to further rule out infectious causes of scleritis was positive. The cloned PCR products were sequenced, but the sequences did not correlate with any specific microbial species. A conjunctival swab of the right eye was negative for aerobic, anaerobic, and fungal organisms. Urine was negative for blastomyces and histoplasma antigen, and serologies were negative for E. canis, R. rickettsia, B. burgdorferi, and B. henselae. Blastomyces and histoplasma agar gel immunodiffusion were both negative and fecal culture for Yersinia pseudotuberculosis was also negative. Based on these findings, an infectious cause of granulomatous scleritis was considered highly unlikely.

The patient was discharged the day after surgery on amoxicillin/clavulanic acid oral suspension, carprofen, and omeprazole. The temperature had returned to normal at $39^{\circ} \mathrm{C}$. At suture removal 10 days later, the surgical site had healed without complication. On subsequent recheck appointments, the patient's left eye exhibited focal scleral thickening and anterior uveitis that was responsive to an immunosuppressive dose of prednisolone ( $2 \mathrm{mg} / \mathrm{kg} / \mathrm{day})$. The patient also developed ecchymoses on his ventral abdomen. The patient had a normal platelet count, no history of trauma, and normal clotting times when these lesions developed. For this reason, the patient was treated with pentoxifylline for presumptive immune-mediated vasculitis.

\section{Conclusions}

Canine idiopathic scleritis is an uncommon diagnosis and has been rarely reported $[12,13]$. This case represents the first case of scleral rupture secondary to non-necrotizing scleritis to be reported in the peer reviewed literature. The possibility of resultant scleral rupture of the globe in these cases makes early diagnosis and aggressive treatment of utmost importance.

A diagnosis of primary scleritis was made as opposed to scleritis secondary to uveal inflammation because the sclera was affected first clinically and was more affected by granulomatous inflammation than the uvea. Histopathologic findings similar to those reported here have been described in other cases of granulomatous scleritis [13]. A designation of idiopathic granulomatous scleritis was made after special stains failed to reveal the presence of microorganisms and systemic infectious disease tests were negative. This case was further categorized as non-necrotizing scleritis because the characteristic hypereosinophilic staining of collagen seen in necrotizing scleritis was absent [14] and because Masson trichrome staining demonstrated separation of collagen by the inflammatory infiltrate. Although it is not common for canine cases of immune-mediated scleritis to be associated with systemic immune-mediated disease, our case appeared to have evidence of systemic disease.

Scleral thinning has been previously associated with necrotic scleritis and scleritis secondary to Ehrlichia canis [12] but antemortem serology in our patient was negative for this agent. Blunt scleral trauma is another cause of scleral rupture. Dogs with scleral rupture secondary to blunt trauma generally present with acute hyphema, subconjunctival hemorrhage, and eyelid or conjunctival swelling [15]. The histological changes seen with blunt trauma include hemorrhage in the anterior and posterior chambers, retinal detachment, choroidal edema, choroidal hemorrhage, and subchoroidal hemorrhage [15]. Our case does not fit with the clinical presentation or histopathologic changes seen with blunt trauma.

Scleral rupture in this case was most likely secondary to scleral thinning and staphyloma formation. The sclera is composed of irregularly arranged collagen and elastic fibers. In scleritis, these fibers are dissected by granulomatous inflammation and in this case the connective tissue fibers were almost completely effaced multifocally. The lack of normal collagen structure creates a weakened focus of sclera where normal intraocular pressure can result in scleral thinning and protrusion of uveal tissue. With severe staphyloma formation, scleral rupture can occur.

Scleral rupture usually results in irreversible vision loss. Cases of scleral rupture secondary to scleritis in dogs have not been well described in the peer-reviewed literature. Surgical repair of these cases is difficult due to the inability of the abnormal sclera to hold suture. Further investigation is warranted to determine the most appropriate treatment and diagnostic recommendation in new cases of scleritis. At this time, it seems most prudent to perform diagnostic tests to rule out infectious causes of scleritis and then institute aggressive immunosuppressive therapy. 


\section{Abbreviations}

OD: Right eye

OS: Left eye

OU: Both eyes.

\section{Authors' Contribution}

Lori J. Best drafted the paper and participated in clinical decision making and case diagnostics. Shelley J. Newman provided interpretation of histopathology and revised paper for appropriate intellectual content. Daniel A. Ward participated in case management and diagnostics and revised paper for appropriate intellectual content. Diane V. H. Hendrix participated in case management, diagnostics, and paper revision.

\section{References}

[1] A. R. Deykin, A. Guandalini, and A. Ratto, "A retrospective histopathologic study of primary episcleral and scleral inflammatory disease in dogs," Veterinary and Comparative Ophthalmology, vol. 7, pp. 245-248, 1997.

[2] D. J. Maggs, "Cornea and sclera," in Slatter's Fundamentals of Veterinary Ophthalmology, D. J. Maggs, P. A. Miller, and R. Ofri, Eds., pp. 175-202, Elsevier Saunders, St. Louis, Mo, USA, 4th edition, 2008.

[3] B. H. Grahn and L. S. Sandmeyer, "Canine episcleritis, nodular episclerokeratitis, sclertitis, and necrotic scleritis," Veterinary Clinics of North America, vol. 38, no. 2, pp. 291-308, 2008.

[4] C. L. Martin, "Cornea and sclera," in Ophthalmic Disease in Veterinary Medicine, C. L. Martin, Ed., pp. 241-297, Manson, London, UK, 2005.

[5] S. C. Reddy and U. R. K. Rao, "Ocular complications of adult rheumatoid arthritis," Rheumatology International, vol. 16, no. 2, pp. 49-52, 1996.

[6] S. L. Harper, E. Letko, C. M. Samson et al., "Wegener's granulomatosis: the relationship between ocular and systemic disease," Journal of Rheumatology, vol. 28, no. 5, pp. 1025-1032, 2001.

[7] A. Heiligenhaus, J. E. Dutt, and C. Stephen Foster, "Histology and immunopathology of systemic lupus erythematosus affecting the conjunctiva," Eye, vol. 10, no. 4, pp. 425-432, 1996.

[8] B. C. Gilger, O. J. Franck, and E. Bentley, "Diseases and surgery of the canine cornea and sclera," in Veterinary Ophthalmology, K. N. Gelatt, Ed., pp. 743-745, Blackwell, Oxford, UK, 2007.

[9] R. R. Dubielzig, K. Ketring, G. J. McLellan, and D. A. Albert, "Granulomatous scleritis, necrotizing scleritis," in Veterinary Ocular Pathology, pp. 232-234, Elsevier, 2010.

[10] R. D. Whitley and B. C. Gilger, "Diseases of the canine cornea and sclera," in Veterinary Ophthalmology, K. N. Gelatt, Ed., pp. 635-671, Lippincott, Wiliams and Wilkins, Philadelphia, Pa, USA, 3rd edition, 1999.

[11] B. H. Grahn and R. L. Peiffer, "Fundamentals of veterinary ophthalmic pathology," in Veterinary Ophthalmology, K. N. Gelatt, Ed., pp. 393-394, Blackwell, Oxford, UK, 2007.

[12] A. A. Komnenou, M. E. Mylonakis, V. Kouti et al., "Ocular manifestations of natural canine monocytic ehrlichiosis (Ehrlichia canis): a retrospective study of 90 cases," Veterinary Ophthalmology, vol. 10, no. 3, pp. 137-142, 2007.
[13] M. J. Day, J. R. B. Mould, and W. J. Carter, "An immunohistochemical investigation of canine idiopathic granulomatous scleritis," Veterinary Ophthalmology, vol. 11, no. 1, pp. 11-17, 2008.

[14] N. Denk, L. S. Sandmeyer, C. C. Lim, B. S. Bauer, and B. H. Grahn, "A retrospective study of the clinical, histological, and immunohistochemical manisfestations of 5 dogs originally diagnosed histologically as necrotizing scleritis," Veterinary Ophthalmology, vol. 15, pp. 102-109, 2012.

[15] A. Rampazzo, C. Eule, S. Speier, P. Grest, and B. Spiess, "Scleral rupture in dogs, cats, and horses," Veterinary Ophthalmology, vol. 9, no. 3, pp. 149-155, 2006. 

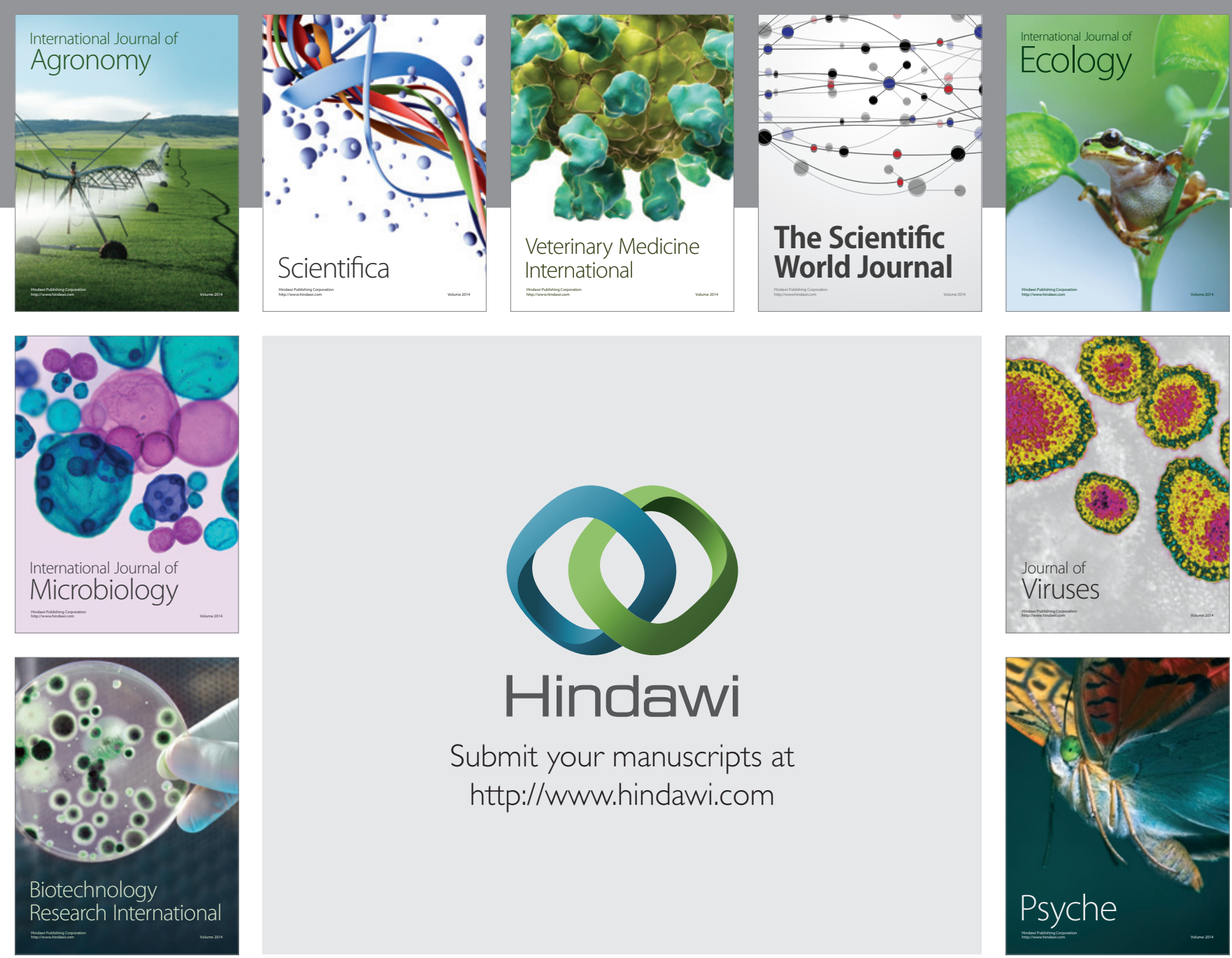

Submit your manuscripts at http://www.hindawi.com
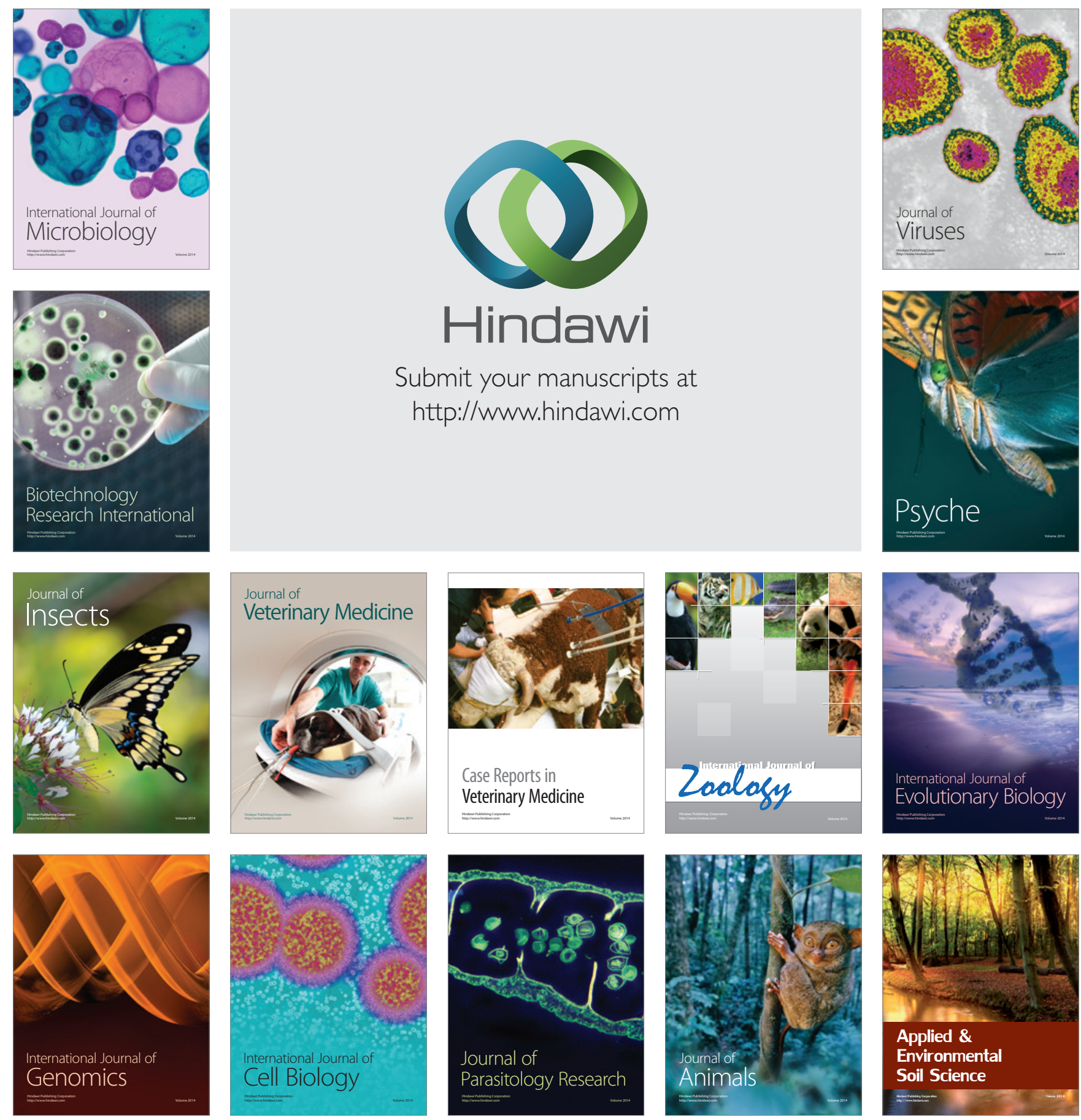